La revue La revue pour l'histoire du CNRS

\title{
Quels statuts pour les chercheurs ? Le débat en
} 1968

\section{Girolamo Ramunni}

\section{(2) OpenEdition \\ Journals}

Édition électronique

URL : https://journals.openedition.org/histoire-cnrs/1350

DOI : 10.4000/histoire-cnrs. 1350

ISSN : 1955-2408

Éditeur

CNRS Éditions

Édition imprimée

Date de publication : 3 mai 2005

ISBN : 978-2-271-06250-5

ISSN : $1298-9800$

\section{Référence électronique}

Girolamo Ramunni, «Quels statuts pour les chercheurs? Le débat en 1968 », La revue pour l'histoire du CNRS [En ligne], 12 I 2005, mis en ligne le 03 mai 2007, consulté le 20 mai 2021. URL : http:// journals.openedition.org/histoire-cnrs/1350; DOI : https://doi.org/10.4000/histoire-cnrs. 1350

Ce document a été généré automatiquement le 20 mai 2021.

Comité pour l'histoire du CNRS 


\title{
Quels statuts pour les chercheurs? Le débat en 1968
}

\author{
Girolamo Ramunni
}

1 Le Comité pour l'histoire du CNRS a organisé, le 17 juin 2004, dans l'auditorium Marie Curie du siège du CNRS, une conférence-débat sur la réforme des statuts des personnels de recherche entrée en vigueur en 1984. Jean-Pierre Chevènement, ministre de la Recherche et de l'Industrie à l'époque a introduit les travaux par son témoignage, suivi de ceux de Jean-Jacques Payan, Pierre Papon, Philippe Lazar et François Kourilsky. Les témoignages des deux secrétaires généraux de l'époque, Philippe Didier et Jean-Marie Bertrand, ont complété la conférence. Tout au long de cette journée, il a été question à plusieurs reprises de replacer ce qui s'est passé en 1984 dans une perspective historique. Pour répondre à cette demande, nous avons essayé de reconstruire le débat sur les statuts en rappelant deux moments clés. Le premier est le colloque de Caen en 1956. L'un des points du programme d'expansion de la recherche dans les dix années suivantes porte sur la nécessité d'un statut pour les chercheurs, en particulier pour ceux du CNRS, alors que l'essentiel des débats a porté sur l'Université, sur l'enseignement supérieur. On peut même dire que les premiers statuts des chercheurs au CNRS, en 1959, sont une conséquence directe de ces débats de Caen, même si cela se concrétise dans un contexte politique qui n'est plus celui de la ive République. Dix ans après cette première étape, la question des statuts est de nouveau d'actualité. Commencé en mars 1968 à l'initiative de la DGRST, amplifié par les événements de mai 1968, ce débat se termine momentanément en décembre 1968 avec le dépôt du rapport de la commission mise en place par la DGRST. Bref, pendant un an, alors que l'actualité semble prendre de court les observateurs attentifs de la société française, la question des statuts revient au cœur des débats dans la communauté des chercheurs. Nous consacrons l'essentiel de cet article à ce qui s'est passé en 1968, car s'amorcent, à cette occasion, des thématiques que l'on retrouvera par la suite, et aujourd'hui encore, quand on évoque la question des statuts. Nous présentons de larges extraits du rapport de la DGRST de décembre 1968 ainsi que des extraits des documents rédigés par les chercheurs, en très grande majorité des chercheurs CNRS, lors des assises de la recherche de juillet 1968 à Orsay. Une question essentielle rejoint celle des statuts : 
dans quelle mesure peut-on concilier le temps d'une carrière attrayante dans la recherche avec le temps propre de la recherche? Cette tension est à notre avis à l'origine de la demande de reconnaissance du métier de chercheur. Pour la résoudre, la distinction entre carrière et fonction est introduite, car, à l'époque du moins, il est reconnu par tous les partenaires sociaux qu'un chercheur pourrait changer de fonction au cours de sa carrière.

Une demande qui naît avec le CNRS

2 Après la guerre, en l'absence d'un statut régulant la carrière de l'ensemble des personnels de recherche, se pose pour la direction du CNRS la question des grilles de rémunération des chercheurs, ingénieurs et administratifs. Faut-il continuer, comme on l'a fait au tout début, à adopter pour le personnel technique les salaires du privé, selon les lieux où sont situés les laboratoires, et pour les chercheurs, celui de l'Éducation nationale? Ce n'est pas possible, car il arrive alors que des chercheurs aient un salaire inférieur à celui des techniciens. Faut-il assimiler les chercheurs aux ingénieurs? Ce qui aurait permis de donner aussi aux chercheurs les salaires du privé. Ce n'est pas le souhait des chercheurs qui, en très grande majorité, ont opté pour l'équivalence avec les carrières de l'enseignement supérieur ${ }^{1}$. Cette question a déjà provoqué quelques grèves, à Bellevue par exemple, au moment du classement des techniciens; de plus, cela soulève les premières protestations de membres du conseil d'administration faisant remarquer la facilité avec laquelle le personnel technique est recruté, à un moment où l'incertitude domine quant à leur statut. Ces épisodes, brièvement rappelés, et qui se passent à un moment où Georges Teissier et Frédéric Joliot collaborent étroitement à la direction du CNRS, montrent non seulement à quel point la question du statut du personnel est présente dès l'origine, mais que déjà sont posées les deux alternatives qui vont dominer la suite des débats: le statut des personnels du CNRS doit-il se conformer au modèle de l'Éducation nationale ou faut-il adopter un modèle de carrière propre au CNRS ? La deuxième solution débouche sur la reconnaissance du métier de chercheur, considéré comme différent de celui d'enseignant-chercheur. L'attitude adoptée dans l'immédiat après-guerre est l'attente. La création du Conseil supérieur de la recherche scientifique, vivement souhaité par les scientifiques, aurait pu apporter une solution. On aurait pu alors envisager une grille commune à tous les organismes de recherche. Mais ce conseil n'est pas créé.

3 La décision de 1945 de reprendre, pour les chercheurs du CNRS, le modèle de l'Éducation nationale pèsera par la suite. De toute manière, en 1945, il est théoriquement possible de faire une carrière entièrement au CNRS, à la condition de voir son engagement régulièrement renouvelé. Pour les techniciens, il faut attendre le décret du 28 mai 1952 qui reprend pour le CNRS le modèle de la fonction publique. On aboutit à une première cohérence, le modèle de la fonction publique ayant été adopté pour l'ensemble du personnel. Cette situation ne parait pas satisfaire les chercheurs qui, à un moment où l'emploi scientifique est en pleine expansion, commencent à réclamer un statut leur garantissant une carrière au CNRS. Lors du premier Colloque de Caen (1er au 3 novembre 1956), le CNRS est mentionné de temps à autre, essentiellement pour rappeler sa place dans le dispositif français de recherche. La nécessité d'un statut pour les personnels, essentiellement pour les chercheurs, est la seule demande explicitement formulée. Le directeur du CNRS, Gaston Dupouy, dans son intervention lors de la séance d'ouverture, parle du danger de l'exode des chercheurs du CNRS vers d'autres organismes dotés d'un statut plus favorable. «La raison est 
claire: les chercheurs du CNRS et de l'Institut national d'hygiène ${ }^{2}$, connaissent le "cumul des désavantages». Sans avoir la stabilité d'emploi des fonctionnaires, ni même toutes leurs indemnités, ils ne peuvent prétendre aux rémunérations offertes par les secteurs privés et semi-publics; et le renouvellement périodique auquel ils sont soumis, donne à leur emploi une précarité qu'on ne rencontre nulle part ailleurs ». Et il va jusqu'à pronostiquer: "S'il n'est pas remédié à cet état de choses, il est malheureusement trop certain que, dans quelques années, le CNRS, tout comme l'Université, sera dépourvu de chercheurs qualifiés et, par là même, mis dans l'impossibilité de remplir sa mission ${ }^{3}$.»

4 La commission chargée de la recherche fondamentale et de l'enseignement scientifique supérieur discute à Caen de ce sujet. Présidée par le député Albert de Balliencourt, le rapporteur général en est André Lichnerowicz, professeur au Collège de France; il s'appuie sur les travaux de la commission préparatoire composée d'Edmond Bauer, professeur honoraire à la Sorbonne, de Jacques Monod, chef de service à l'Institut Pasteur et de lui-même. Des communications sont présentées par Charles Sadron, Marcel Prettre, Alfred Kastler et Étienne Wolf. Dans le rapport introductif, il est fait référence au CNRS et on y trouve des propos qui rappellent ceux de Gaston Dupouy, soulignant les tensions qui peuvent se créer au sein d'un laboratoire entre chercheurs CNRS et universitaires. Quant aux statuts, il en est demandé un qui « en respectant leur [du CNRS] originalité propre et sans les identifier au personnel de l'Enseignement supérieur, leur assure les mêmes avantages matériels, la même indépendance, la même stabilité, les mêmes garanties morales [...]. L'identité matérielle et morale des postes du CNRS avec ceux de l'Enseignement supérieur permettrait au personnel du CNRS d'être appelé par les facultés à participer, à égalité d'autorité avec le personnel de l'Enseignement supérieur, à la formation des chercheurs et tout particulièrement à la direction des chercheurs du $3^{e}$ cycle et à l'organisation de cet enseignement. ${ }^{4}$ » Le reste du rapport est essentiellement consacré à l'enseignement supérieur. Ce qui n'est pas étonnant car la majorité des participants sont des universitaires. Des idées semblables seront reprises par Charles Sadron qui, dans sa contribution, va jusqu'à prévoir dans les futurs statuts que les chercheurs consacrent « obligatoirement, deux heures de service par semaine, à la faculté du lieu où réside leur centre de recherches. Ces heures seront mises à la disposition du doyen qui en disposera après entente avec le directeur du CNRS. » La seule voix discordante est celle de Raymond Dedonder, à l'époque chargé de recherches au CNRS. Le 18 mars 1956, avec l'approbation d'Alfred Kastler, a été constitué le Syndicat national des chercheurs scientifiques ; il est né, selon les acteurs, de la nécessité de défendre la carrière des chercheurs au CNRS. Raymond Dedonder fait partie du bureau du syndicat dès l'origine. Lors de son intervention à Caen, il fait remarquer que « dans l'état actuel de l'organisation universitaire, la participation du personnel CNRS à l'enseignement n'est pas souhaitable, et qu'elle a lieu, trop souvent dans des conditions défavorables, pour ce personnel comme pour l'enseignement luimême. »

5 Un texte final fixe les priorités à réaliser dans les dix années suivantes; il ne sera pas fait état de ces divergences. Le cinquième point de cette déclaration porte sur la nécessité d'un statut du personnel du CNRS: «L'un des points les plus urgents du programme d'expansion doit donc être l'élaboration d'un statut valable pour ce personnel. Ce statut devrait respecter l'originalité propre du cadre du CNRS et, sans l'identifier aux cadres fonctionnarisés de l'Enseignement supérieur, assurer à ses membres - après une période probatoire convenable - les avantages de stabilité et 
d'indépendance, ainsi que les garanties morales dont jouit le personnel de l'Enseignement supérieur. Ce statut doit être établi en vue d'une harmonisation de la condition des chercheurs dans tous les organismes publics. »

6 Le décret de $1959^{5}$ sur les statuts réalise une partie de ce vœu. Certains acteurs de Caen ont participé à leur rédaction en tant que membres du Comité consultatif de la recherche scientifique et technique. Jean Coulomb, absent excusé à Caen, a participé à l'élaboration des statuts en tant que directeur général du CNRS et présidé à leur mise en place. Se termine alors une première phase de la question des statuts. Reste à réaliser ce qui est indiqué par la dernière phrase du vœu de Caen : l'harmonisation des statuts des chercheurs de l'ensemble des organismes publics de recherche. Mais pour que cela soit possible, il a fallu le travail important du CCRST, conduisant à la réforme des autres organismes de recherche ainsi qu'à la vision unitaire du monde de la recherche par le biais de la préparation annuelle de l'enveloppe recherche. Ce n'est pas étonnant que ce soit au sein du CCRST qu'est revenue la question des statuts et de leur indispensable harmonisation.

Le point de vue du CCRST et de la DGRST

7 " Le Comité consultatif de la recherche scientifique et technique a constitué en son sein en 1967 plusieurs groupes de travail dont l'un, sous la direction de Robert Chabbal, a été consacré aux problèmes des hommes et des chercheurs. Ce groupe a élaboré en mars 1968 l'un des rapports soumis au Comité interministériel de la recherche d'avril 1968. Ce document, après avoir présenté le problème sous son aspect quantitatif, examinait les conditions dont dépendait l'efficience des chercheurs: formation, mobilité, «management ». Pour ce qui est du second point, ce rapport dégageait quatre types de mobilité :

- celle qui conduit le chercheur à changer de sujet ou de technique sans changer de secteur ni de type de recherche ;

- le passage d'un type de recherche à un autre type de recherche ;

- passage de la recherche à d'autres activités (développement et productiontechnico-commercial)

- passage du secteur public au secteur privé et vice versa.»

8 Il proposait déjà quelques mesures mais insistait surtout sur la finalité de la mobilité : assurer, dans l'intérêt des individus et de l'économie en général, une utilisation optimale des chercheurs. Sur l'intérêt d'une mobilité des chercheurs pour le développement de l'économie nationale, le rapport poursuivait en ces termes: «le groupe a pourtant insisté sur l'intérêt fondamental de ces passages d'un secteur à l'autre: diffusion des techniques, dialogues considérablement facilités entre la recherche fondamentale et la recherche industrielle. Il a été constaté certains progrès et tout d'abord le simple fait que de larges secteurs de l'opinion industrielle et universitaire soient fondamentalement d'accord sur la nécessité de ces échanges. Le groupe estime cependant que, pour faciliter cette mobilité, des mesures spectaculaires doivent être prises. Ces dernières, pour être efficaces, devront d'ailleurs tenir compte du milieu où elles seront appliquées : dans une société où la sécurité d'emploi est le plus recherché des avantages, il serait injuste et d'ailleurs sans effet de condamner le seul chercheur à de fréquents changements d'emplois [...]. Il faut cependant insister sur les dangers que présente une trop grande mobilité. Loin d'être une panacée, la mobilité peut vite devenir destructive. Nombreux sont les exemples de chercheurs totalement inefficaces parce que leur centre d'intérêt se déplace perpétuellement, d'équipes qui au contraire doivent une part de leur succès à leur ténacité et à la stabilité de leurs cadres. 
Il faut au moins cinq ans, et même souvent dix ans, pour former un chercheur réellement utilisable dans un secteur avancé. Méconnaître un tel fait revient à sacrifier la réalité à de la stratégie en chambre ${ }^{6}$. »

Cette urgence est non seulement le fait de la volonté des scientifiques d'avancer dans la réalisation de ce qui avait été l'un des vœux de Caen en 1956, mais aussi, et probablement surtout, de la comparaison qui pouvait être faite entre la mobilité des chercheurs français et de celle des chercheurs étrangers. C'est le résultat d'études menées au sein de l'OCDE sur les politiques nationales de recherche. Le rapport cite en effet l'étude du sociologue et historien des sciences Joseph Ben David qui avait présenté ses idées sur la nécessaire mobilité au sein du monde de la recherche à la conférence internationale sur la science des pays de l'OCDE (11-12 mars 1968). "Ouvrir les institutions scientifiques d'un pays aux influences extérieures et accepter le principe de la mobilité scientifique internationale n'a pas seulement l'avantage d'éliminer les inconvénients du particularisme local et de l'exiguïté du marché; il est en outre probable qu'il en résultera un effet favorable à la créativité... Plus les liens entre les savants d'un pays et leurs collègues, compatriotes ou étrangers, qui travaillent dans les mêmes domaines ou dans des domaines apparentés, seront étroits, plus le niveau de l'activité scientifique de ce pays sera élevé... Même dans leur propre pays, toutes sortes de barrières s'opposent aux mouvements des scientifiques. Parfois, leur passage d'une institution à une autre du même genre (d'une université ou d'un institut public de recherche à d'autres) se trouve limité ou étroitement circonscrit. Mais, dès lors qu'il s'agit de mutations entre des établissements de types différents, entre l'université et l'industrie ou la recherche publique (et inversement), la mobilité est réduite dans tous les pays d'Europe. » Si la mobilité interne est à cette époque le mal commun des pays européens, en ce qui concerne la France ce sont aussi les échanges internationaux qui semblent faire problème. Le tableau ci-dessous montre bien la situation.

Pour étudier les causes de ce comportement et imaginer des solutions au niveau des statuts, Maurice Schumann, ministre d'état chargé de la recherche scientifique et des questions atomiques et spatiale, décide le 16 mars 1968 :

11 «Article $1^{\mathrm{er}}$ - Il est créé un groupe de travail ayant pour tâche d'étudier les problèmes de la carrière des chercheurs et leur mobilité.

12 Article 2 - Ce groupe de travail devra déposer un rapport le $1^{\text {er }}$ juin 1968 pour présentation au Comité interministériel de la recherche scientifique et technique.

13 Article 3 - Ce groupe de travail, présidé par le Délégué général à la recherche scientifique et technique, assisté du secrétaire général et du conseiller juridique de la Délégation générale, comprend :

- un représentant du ministre d'État chargé de la fonction publique

- un représentant du ministre d'état chargé de la recherche scientifique et des questions atomiques et spatiales

- un représentant du ministère de l'Économie et des Finances

Source : DGRST, 1967

- un représentant du ministre de l'Éducation nationale

- Robert Chabbal, professeur à la faculté des sciences d'Orsay

- André Danzin, vice-président directeur général de la CSF

- Jules Horowitz, directeur des piles atomiques au CEA

- Claude Lasry, conseiller d'État

- Lucien Malavard, professeur à la faculté des sciences de Paris 

générale. Le groupe de travail pourra procéder aux auditions nécessaires, en particulier des syndicats concernés. recherche scientifique et technique du 26 avril 1968. Le Comité accorde un délai supplémentaire au groupe de travail, posant la fin de l'année comme date limite pour la présentation de leur rapport.

Le groupe de travail a été constitué quelques jours avant l'occupation de la salle du conseil de la faculté des lettres de Nanterre par des étudiants, qui créent le mouvement du 22 mars, alors que selon Pierre Viansson-Ponté, dans son billet hebdomadaire du Monde, la France s'ennuie. Le 2 mai, la faculté de Nanterre est fermée et le lendemain ont lieu les premiers affrontements entre les étudiants, qui se sont installés à la Sorbonne, et la police. C'est le prélude à la «nuit des barricades (11-12 mai) » et au défilé de la République à Denfert-Rochereau, rassemblant étudiants et syndicalistes (13 mai). Le lendemain, les grèves se généralisent dans les laboratoires du CNRS. Tout est mis en discussion : structures de recherche, conditions de réalisation des programmes, promotion personnelle "des travailleurs scientifiques », dénomination utilisées dans les documents à la place de chercheurs et/ou de techniciens. Des groupes de discussion s'installent dans les grands centres de recherche comme Bellevue, la Halle aux Vins et l'Observatoire de Meudon. Dans le même temps, des chercheurs s'installent au siège du CNRS où un Comité d'action central rassemblant des représentants de différents laboratoires organise un début de coordination dans un flot invraisemblable de documents le plus divers. Alors que des élections législatives se préparent après la dissolution de l'Assemblée le 30 mai par le général de Gaulle, le Comité d'action central $\mathrm{du}$ CNRS, avec l'accord de certains syndicats ${ }^{7}$, propose le 3 juin la réunion d'une conférence nationale. Un bureau est constitué, composé par : Robert Klapisch, Comité d'action du CNRS, P. Leroux-Hugon, syndicat national des chercheurs scientifiques (Snes-Fen), A. Salin, syndicat général de l'Éducation nationale (Sgen-CFDT), M. Taieb, syndicat national indépendant de la recherche scientifique (Snirs-CGC). L'appel lancé pour une conférence nationale des travailleurs de la recherche scientifique indique les objectifs : «Limitée dans un premier temps aux secteurs de «l'enveloppe » (CNRS, Inra, Inserm, Orstom), la conférence permettra d'abord de faire la synthèse des travaux effectués dans les laboratoires et les différentes commissions et de dégager un large accord sur les nouvelles perspectives de la recherche dans ces secteurs [...]. Orientée d'abord vers la définition de nouvelles structures, la réflexion s'est élargie jusqu'à remettre en cause la place de la recherche dans la société, ses relations avec l'Université et les autres secteurs. Finalement, les travaux de la conférence seront regroupés en cinq thèmes :

1) Politique et financement de la recherche au niveau national.

2) Organisation et gestion de la recherche.

3) Statut des personnels de la recherche.

4) Relation recherche Université.

5) La recherche et ses applications. ${ }^{8}$ "

La revue pour l'histoire du CNRS, 12 | 2005 
19 Le principe d'organisation de la conférence est simple : 1 délégué pour 40 chercheurs et 1 pour 80 techniciens. Les petits laboratoires sont invités à se regrouper afin d'envoyer des délégués. Du 10 au 12 juillet 1968, à la faculté des sciences d'Orsay, 355 délégués élus par 11000 travailleurs scientifiques (sur un effectif total de 20000 ) et 200 invités se réunissent. Parmi les invités, il y a aussi le Délégué général à la recherche scientifique et technique, Pierre Aigrain, qui participe au moins aux séances plénières. Sur les 355 délégués, 291 avaient été élus par le CNRS, 15 par l'Orstom, 19 par l'Inra et 30 par l'Inserm. Globalement, deux tiers des délégués représentaient les chercheurs et un tiers les personnels administratifs et techniques. Le rapport introductif aux travaux concernant le statut des personnels est présenté par L. Gatineau et G. Hartmann. Les débats sur ce sujet se déroulent en deux sous-commissions tout au long de la journée du 11 juillet. C'est en séance plénière, le 12 juillet après-midi, que le texte du rapport final est discuté et approuvé. Les points essentiels sont les suivants :

En résumé, on peut dire que de la conférence d'Orsay, premières assises de la recherche animées essentiellement par les chercheurs du CNRS, une position claire se dégage : le préalable à toute mobilité intra et inter-organismes est la titularisation dans un corps qui garantisse les avantages sociaux accordés aux titulaires de la Fonction publique. Le groupe de travail institué juste avant les événements de mai 1968 peut-il négliger ces demandes émanant du monde de la recherche? Quelles modifications sont alors apportées à leurs suggestions?

Retour au groupe de travail de la DGRST

21 À la rentrée, le groupe procède à des auditions. Le 15 octobre, c'est au tour des directeurs des organismes de recherche. Ensuite, ce sera le tour des syndicats. Les différences qui se dégagent minent l'idée d'un corps unique de l'État. Pour Eugène Aujaleu, «le mouvement de l'Inserm vers l'extérieur concerne les femmes qui abandonnent leur carrière et les personnels qui embrassent une carrière hospitalouniversitaire [...]. Il est souhaitable de parvenir, dans le futur, à un recrutement commun pour l'Inserm et les carrières hospitalo-universitaires. " Il se déclare peu favorable à l'institution d'un corps filet. Jacquard, de l'Ined, est lui aussi réservé sur le principe du corps filet. En ce qui concerne son organisme (40 chercheurs), il n'y a pas de problèmes de mobilité. Pour Guy Camus, de l'Orstom, « le problème qui se pose n'est pas d'inciter à la mobilité mais de freiner les tendances au détachement dans les universités d'outre-mer, l'Inra, les sociétés de développement, ceci afin de conserver un encadrement suffisant des équipes de laboratoires [...]. En ce qui concerne le corps filet, il appelle [...] deux types de remarques. Sur le plan général, il n'est pas d'accord avec la philosophie du projet. Il estime qu'au bout de cinq ans, le corps sera engorgé par des chercheurs peu utilisables et, par là, mettra en évidence le nombre d'échecs. D'autre part, l'affectation autoritaire pèsera sur le recrutement des organismes et risque de diminuer le niveau de l'enseignement par la mise en poste de personnels aigris. Il ne pense pas que le corps filet règlera la situation. » Pour Pascal, du CEA, « la mobilité est insuffisante. Quelques départs vers l'industrie commencent à se développer mais en nombre trop faible... Le corps filet pose de graves problèmes de fonctionnement : il ne peut être équilibré que si les besoins des organismes sont compensés par des recyclages facilitant le changement de fonctions, ce qui implique nombre de hasards. Il risque de devenir une caisse de secours ou un bureau de placement alors qu'il ne remplirait sa fonction que s'il était vide. À défaut d'autres solutions l'harmonisation générale des statuts faciliterait le règlement des problèmes." Vladimir Mercouroff présente la 
position de l'Enseignement supérieur: "Il ne faut pas oublier toutefois, que tout chercheur ne fait pas un bon enseignant et qu'il existe des éléments de niveau inférieur. À leur égard, le corps filet ne parait pas la bonne solution. La mobilité est insuffisante dans l'Enseignement supérieur [...]. Pour la faciliter, on pourrait retenir : l'harmonisation des statuts, le règlement de la question des retraites et peut-être un corps filet pour les techniciens." Les chercheurs de l'Inra étant à l'époque déjà titulaires, pour le directeur, Février, la position est simple : «Actuellement, la mobilité est freinée par la direction de l'organisme en fonction des besoins d'encadrement des équipes... Il estime que la création du corps filet marquerait la fin du recrutement de l'Inra par la compétition de l'Igref (Ingénieurs du génie rural, des eaux et des forêts) et la multiplication des candidatures de polytechniciens. Il pense que la création d'un corps unique, avec des problèmes de gestion bien difficiles, est en contradiction avec les tentatives actuelles de décentraliser la décision. Il dit ses craintes envers le passage dans l'enseignement, le manque de qualités étant, là, plus grave que dans la recherche... Il suggère que soient étudiées des mesures permettant d'améliorer dans les statuts actuels le détachement dans le secteur privé et rappelle la mesure qui existe dans un corps - l'armée - où le vieillissement est rapide : la retraite anticipée. » La position de Pierre Jacquinot, directeur général du CNRS, est bien différente : «Au CNRS, la mobilité est insuffisante: les spécialités les plus diverses y existent, certaines n'ont pas de débouchés s'il n'y a pas de reconversion, reconversion qui se heurte aux préjugés des utilisateurs - chercheurs non utilisables - et des chercheurs - sentiment de déchéance. Il estime, en ce qui concerne le corps filet, que l'on ne doit pas entacher de suspicion la recherche de garanties manifestée par les personnels. Il rappelle que c'est le rôle joué, pour beaucoup, par l'agrégation. Le but à atteindre étant la mobilité, le corps filet facilite le passage et entraîne l'obligation de trouver une affectation au retour. Il a été inventé pour favoriser le mouvement et éviter la fonctionnarisation totale [...]. Il pense enfin que la mobilité entre fonction enseignante et CNRS devrait être conçue selon le principe des possibilités de passage pendant de longues périodes - 4 à 5 ans - d'un secteur à l'autre. »

Il est fort probable que c'est au sein du ministère de l'Enseignement supérieur que cette idée du corps filet a été conçue pour répondre à la demande, exprimée en juillet par les chercheurs, d'être titularisés dans le cadre de la fonction publique. En effet, on prépare au sein du ministère de l'Enseignement supérieur la loi d'orientation pour l'université. Les premiers textes prévoient "que les établissements pourront recruter, outre les personnels titulaires, des personnels contractuels. Il faut attendre pour connaître la proportion entre les deux catégories, mais il paraît y avoir là un élément de solution au passage de contractuels du CNRS dans l'Enseignement supérieur. " C'est ce que fait remarquer Polverini en fin d'audition des responsables des organismes de recherche. Il s'agit d'harmoniser les situations au CNRS et dans l'Enseignement supérieur, de sorte à faciliter la mobilité entre chercheurs et enseignants-chercheurs. On voit bien que la question des statuts et de la mobilité est dominée par la situation du CNRS et de l'enseignement supérieur. La loi d'orientation de l'enseignement supérieur votée le 12 novembre 1968 prévoit à l'article 30 : «Dans les établissements publics à caractère scientifique et culturel relevant du ministère de l'Éducation nationale, l'enseignement est assuré par des personnels de l'État, des enseignants associés et par des personnels contractuels propres à ces établissements. Ces établissements peuvent faire appel, pour l'enseignement, aux chercheurs, à des personnalités extérieures et, éventuellement, aux étudiants qualifiés. En dérogation au statut général de la Fonction publique, les 
enseignants de nationalité étrangère peuvent, dans les conditions fixées par un décret en Conseil d'État, être nommés dans les corps d'enseignants de l'Enseignement supérieur."

Un corps pour favoriser la mobilité : le corps filet

Le corps filet a été imaginé pour donner une compensation à ceux qui, titulaires dans un corps à créer de chercheurs, feraient preuve de mobilité. Il s'agit, en échange de la mobilité, d'accorder aux chercheurs la stabilité de l'emploi par l'affiliation au régime de la Fonction publique (titularisation). Le groupe de travail est conscient que son efficacité dépend de sa généralisation à tous les organismes. Ce qui signifie que pour les organismes où les chercheurs sont à l'époque déjà titulaires, il fallait créer un corps de contractuels, généralisant ainsi à l'ensemble des organismes de recherche, et de l'Enseignement supérieur aussi, comme on l'a vu, la possibilité d'une double carrière : contractuels et titulaires. Les objections d'ordre réglementaires sont nombreuses, surtout si l'on veut soumettre la carrière dans le corps filet à certaines conditions n'existant pas dans les autres corps de la Fonction publique. Le groupe est conscient des problèmes qui se posent en ce qui concerne l'entrée dans le corps. Les règles de la Fonction publique ne prévoient pas la nomination pour ordre. «Or, il est patent que les nominations dans le corps filet proposé seraient destinées non à pourvoir à des emplois, mais à faciliter le recyclage de chercheurs ayant perdu leurs facultés d'invention, à atténuer les conséquences sociales d'un certain chômage frictionnel. Le principe d'un corps axé sur une autre notion que la notion d'emploi est évidemment dérogatoire aux règles de la fonction publique. Mais c'est toute la philosophie du système qui est ici en cause, et on peut espérer que si le dispositif envisagé correspond à un besoin et que ce besoin est admis, les instances juridiques (Conseil supérieur de la Fonction publique, Conseil d'État) toléreront la dérogation. Toutefois, pour la portée du précédent qui serait ainsi créé, il conviendrait d'apporter un soin extrême à la présentation du caractère particulier de ce corps. »

La création de ce nouveau corps exige aussi des dérogations quant aux concours d'entrée. Le concours sur épreuves doit être écarté car non viable pour ce type d'emplois. D'autre part, les jurys devraient être organisés à l'intérieur du nouveau corps, ce qui assurerait leur autonomie par rapport aux organismes, tout en prenant en compte l'évaluation sur les aptitudes des candidats, faite au sein des organismes où ils auraient effectué leur stage, par exemple. «Il convient de trouver un système qui, en tenant compte de l'avis des instances de recherche (commissions du CNRS par exemple), situe le jury du concours auprès du chef du corps filet. Cette procédure doit être institutionnelle car il n'est pas possible de laisser aux simples rapports de bon voisinage, à la valeur des contacts humains que le chef du corps des chercheurs d'État entretiendra sans nul doute avec les directeurs des établissements de recherche, le soin de décider des titularisation dans le corps d'État. Elle pourrait consister à reconnaître aux commissions des organismes un rôle de pré-jury qui aurait à classer les candidats, la décision incombant à un jury unique au niveau du corps. " Comment déterminer chaque année le nombre des agents admis dans le corps? "Le nombre d'emplois disponibles chaque année est égal à la somme des emplois disponibles dans les instituts de recherche (création d'emplois et emplois libérés par les départs volontaires et contraints).

Deux modalités sont possibles :

a) système de la liste d'aptitude permettant de pourvoir aux vacances au fur et à mesure qu'elles se présentent, 
b) système du concours valable pour un an avec un nombre de postes limité, fixé au début de chaque année. " inflationniste, alors que c'est exactement le contraire que l'on cherche. Une autre question concerne la détermination des effectifs dans le corps : «Les avantages d'un corps aux effectifs limités sont évidents : seule cette circonstance permet de limiter et de connaître la charge financière du corps (retraites notamment) et de provoquer des incitations à une gestion ferme par le chef de corps (recherche systématique d'intégration dans les corps d'accueil ; détachements d'office, évictions, etc...) Mais la difficulté de fixer les effectifs est la suivante : pour éliminer des chercheurs médiocres et faciliter tous les types de mobilité, il semble qu'il soit nécessaire de pouvoir renvoyer avec aisance les chercheurs dans le corps filet. Or, à l'heure actuelle, il faut, en droit, attendre une vacance pour pouvoir bénéficier d'une réintégration dans le corps d'origine. Il faut donc supprimer ce temps d'attente mais aussi écarter les difficultés qui risquent de surgir si plusieurs organismes veulent, en même temps, mettre fin aux détachements de certains de leurs chercheurs et qui poseront alors la question de savoir dans quel ordre devra se faire la réintégration. La solution de la nomination en surnombre pour régler temporairement ces difficultés ne peut être recommandée en raison des tentations qu'elle comporte. C'est pourquoi il est apparu au groupe de travail, malgré les inconvénients pratiques de la proposition et son caractère très dérogatoire, qu'il était plus raisonnable de préconiser un corps dont les effectifs sont susceptibles de variations annuelles. »

Certes, le corps filet a, aux yeux des concepteurs, l'avantage de rendre plus simples les mobilités dans le privé tout comme le détachement dans un autre corps, et de pouvoir prononcer sous certaine condition "l'expulsion autoritaire, lorsque certaines conditions sont remplies [...]. La possibilité de mise à la retraite d'office à partir de 55 ans avec jouissance immédiate de la pension de retraite ou mise d'office, à partir de cet âge, en position de demi traitement. " Toutes ces conditions particulières et dérogations à obtenir transforment une idée simple sur le papier en un système complexe, dont la gestion n'apparaissait plus si simple. Et pourtant, les objectifs du corps filet que le rapport rappelle dans ses principes, semblent obtenir un large consensus. «La philosophie qui inspire le système du corps filet est simple : il s'agit de promouvoir, et plus encore, d'imposer la mobilité aux chercheurs en contrepartie de quoi leur serait accordée la stabilité de l'emploi sous la forme d'une affiliation aux règles de la Fonction publique. En même temps, on retient l'idée que le passage dans des activités de recherche valorise l'individu et le prépare à exercer utilement d'autres fonctions dans la société, fonctions de production, conseil, enseignement, administration, documentation; cette observation était vraie non seulement pour les chercheurs brillants qui pourraient poursuivre leur carrière entière dans la recherche, mais aussi pour ceux qui, passées les premières années, auraient souhaité se consacrer à d'autres activités. En créant un corps de travailleurs scientifiques pourvus d'un statut de fonctionnaires titulaires, on accordait une sécurité de nature à tranquilliser les esprits, mais en même temps, on appliquait l'une des règles fondamentales de la Fonction publique, celle de l'affectation aux fonctions. Le corps filet aurait permis au chef du corps de prononcer des affectations autoritaires dans d'autres fonctions que celles de la recherche, avec l'espoir que des intégrations dans d'autres emplois de la Fonction publique résorberaient progressivement la masse des chercheurs originaires $\mathrm{du}$ corps filet. Parallèlement, et pour échapper à l'affectation autoritaire, les 
chercheurs appartenant au corps filet auraient été incités à rechercher systématiquement des détachements dans les carrières de leur choix, et notamment sur les emplois, titulaires ou contractuels, des établissements de recherche publics; on escomptait ainsi que le corps filet n'aurait été qu'une voie de passage assez courte, les titulaires étant soit en position de détachement à l'extérieur, soit, pour ceux qui n'auraient pu parvenir à se faire accueillir dans un autre organisme de recherche dans la fonction publique, ou dans le secteur privé, affectés d'office par le chef de corps. Les emplois créés pour ce nouveau corps auraient, dans cette perspective, permis à la fois le recrutement des personnels chercheurs des organismes de recherche actuels et, dès lors qu'on souhaitait pratiquer une politique systématique de détachements et d'affectations d'office, les reconversions des chercheurs entre deux positions à l'extérieur. Pour les moins aptes à la mobilité, le corps filet offrait, en cas d'échec au dehors, une situation minimum ; les autres n'y seraient restés que le temps nécessaire au déroulement de la procédure de recrutement ou au passage d'un secteur à l'autre. Les instigateurs du projet comptaient bien plus sur un vaste courant de détachements volontaires que sur la procédure autoritaire de l'affectation pour faire bouger les chercheurs ; par comparaison avec les avantages de carrière des corps de détachement, la situation dans le corps filet ne devait pas être trop confortable: la courbe de la carrière devait donc avoir un profil très aplati; à titre d'indication, l'une des hypothèses retenues pour fixer l'échelonnement indiciaire avait été de faire parcourir en 35 ans aux personnels du corps filet la partie de la carrière qu'un chercheur du CNRS ou de l'Inserm ne met que 15 ans à franchir à un rythme d'avancement normal. »

Le projet, comme on l'a déjà vu, est mal accueilli par les directeurs des organismes de recherche, mis à part le CNRS et, en partie, le CEA. Le refus des syndicats est net. « Dans l'hostilité des syndicats, qui n'ont cependant pas contesté la nécessité d'une certaine circulation des chercheurs, on a cru pouvoir déceler des motifs divers, pas toujours avoués (craintes d'un autoritarisme excessif, crainte d'inefficacité, affirmation que les incitations au départ vers le secteur privé ne résoudraient pas, sur le plan quantitatif, le problème de la mobilité des chercheurs, la solution étant plutôt dans l'évasion vers l'enseignement supérieur) [...]. Dans l'ensemble, les syndicats ont plutôt saisi l'occasion de la consultation sur les problèmes de la mobilité pour exposer leurs revendications de carrière [...]. Au terme de son enquête, le groupe de travail a dressé le bilan prévisible $\mathrm{du}$ corps filet. Au tableau des avantages, il a fait figurer un certain nombre de conclusions : il lui est apparu que l'adoption du système concourrait certainement à créer des habitudes, des comportements favorables à la circulation des chercheurs; elle permettrait d'imposer, dans le secteur public, une mobilité réelle, y compris une mobilité de fonctions ; un tel dispositif assurerait, plus que la juxtaposition des régimes actuels, une plus grande souplesse des emplois de détachement. De toute manière, il eût été, à des titres divers, plus économique qu'une formule généralisée de fonctionnarisation classique - qui reste l'une des revendications fondamentales des syndicats - et eût permis de s'acheminer progressivement vers la transformation des statuts des titulaires dont bénéficient certains corps de chercheurs en statuts de contractuels. »Certes, il y avait des difficultés d'ordre réglementaire à surmonter, mais ce qui avait le plus frappé les membres du groupe était d'avoir découvert les "particularismes des instituts de recherche et la résistance psychologique des corps qui auraient été sans doute désignés, de manière permanente, comme corps de débouchés. » 
29 En résumé, on voit bien la situation qui s'est créée au fil du temps : la question du statut ne peut pas être discutée uniquement pour le CNRS mais doit prendre en compte la situation de tous les organismes de recherche publics. L'objectif est de trouver des solutions plus simples aux questions de fond auxquelles le groupe avait espéré apporter une solution.

Quelles solutions adopter?

30 La seule marge de manœuvre restante est d'adopter des mesures incitatives pour faciliter la mobilité des chercheurs. La création d'une direction de l'emploi scientifique est suggérée comme mesure préalable, "une sorte de gouvernement de l'emploi scientifique [...] une structure permanente... située auprès du ministre de la Recherche » ayant la responsabilité de « mettre en œuvre les moyens propres à assurer la formation continue des personnels de recherche, y compris le recyclage et la reconversion des chercheurs qu'il y aurait intérêt à orienter vers d'autres fonctions ; de rassembler au sein d'un service, qui pourrait être une sorte de bourse de l'emploi, les informations sur les postes de recherche offerts dans les divers secteurs et en assurer la diffusion systématique; d'informer très largement les secteurs de l'industrie privée, notamment des petites et moyennes entreprises souvent à l'écart des circuits de réflexion et d'information concernant la recherche, de la valeur des personnels formés dans la recherche et de l'intérêt qu'il y aurait à y faire largement appel pour des postes de responsabilité dans les fonctions de production ou technico-commerciales. » Parmi les mesures souhaitées en vue de modifier les comportements des chercheurs face à la mobilité jugée indispensable, le groupe de travail propose que "au moins après sa soutenance de thèse, et même plusieurs fois au cours de sa carrière dans certaines limites, le candidat à une fonction de recherche soit invité à aller passer un an dans un pays étranger ou dans un autre laboratoire de nature et d'implantation très différentes [...].» En ce qui concerne les statuts, le groupe souhaite que des mesures soient prises pour abolir les obstacles à la mobilité, en assouplissant les conditions du détachement et son renouvellement. Ces mesures sont pensées tant pour le secteur public que pour le secteur privé. La mobilité du secteur privé vers le secteur public est aussi considérée comme indispensable. D'où la suggestion : «aux fins d'intégration dans les corps de la fonction publique, d'un tour extérieur à un niveau élevé [...]. L’idée serait [pour faciliter ces passages] qu'au moment du passage dans le secteur public, il soit tenu compte du temps passé dans le privé, à des activités de recherche, pour fixer l'échelon d'intégration et reconstituer les droits à pension de retraite. »

31 Reste ce qui apparemment aurait dû poser le moins de problèmes, la mobilité au sein du secteur public. Et pourtant, dans ce cadre aussi il y a des obstacles à éliminer. « Le groupe de travail a donné ici [le rapport] un catalogue de propositions, dont certaines ont déjà été présentées depuis des années, sans succès. Il insiste sur le fait que l'exigence de mobilité est devenue une nécessité si fondamentale au développement harmonieux de l'économie, qu'un dispositif de grande efficacité doit être mis en place [...]. C'est ainsi que le groupe de travail a pris à son compte la demande déjà formulée de l'année sabbatique pour les membres de l'Enseignement supérieur, cette faculté existant déjà au CNRS à la faveur de la disposition qui permet d'affecter des chercheurs dans les laboratoires privés [...]. Le groupe de travail préconise un assouplissement de la règle, actuellement en vigueur dans les statuts de certains organismes de recherche, qui limite à un faible nombre d'heures la possibilité de participer à des activités externes à la recherche. Plus nouvelle est sans doute la proposition de réserver après 
recyclage, un certain pourcentage des emplois créés dans les organismes de recherche existants [...]. Pour les organismes de recherche dont le personnel est soumis à un statut de contractuel, il est proposé, si pour d'autres raisons il est opportun de s'engager dans la voie de la titularisation, de réserver le bénéfice du statut de la fonction publique aux chercheurs parvenus à un certain niveau et parmi ceux-ci, à ceux qui auraient déjà « fait leur mobilité » selon une expression entrée dans le vocabulaire depuis la réforme du statut des administrateurs civils. C'est ainsi qu'il pourrait être envisagé, au CNRS, de ne laisser les chargés de recherche ayant moins de 10 ans de service accéder au grade de maître de recherche qu'à la condition que les promouvables aient accompli une certaine mobilité. Un dispositif limitant à un certain pourcentage du nombre total d'emplois l'impossibilité de mobilité atténuerait ce que cette mesure pourrait avoir de trop radical. Le cas de l'Inserm mérite une solution également particulière : le personnel de recherche appartenant à l'Inserm devrait voir s'ouvrir les carrières de soins et d'enseignement du secteur public. Enfin, reprenant ici la proposition de certains syndicats, le groupe de travail estime qu'il faudrait s'engager dans une étude minutieuse tendant, sur divers points, à harmoniser les statuts entre organismes de recherche et de ces organismes avec l'enseignement supérieur. Quelques indications sont ici données qu'il faudrait compléter ou soumettre à l'épreuve d'un examen approfondi :

- en début de carrière, autour des notions de stage et de formation à la recherche, les questions de la durée de l'apprentissage requis, des titres susceptibles de sanctionner l'aptitude à la recherche, des indices de traitement, le rapprochement des statuts des divers organismes de recherche pourrait d'abord être envisagé ;

- en cours de carrière, un parallèle plus rigoureux est également recommandé entre l'échelonnement indiciaire du corps de l'Enseignement supérieur et celui des organismes de recherche. Cette observation vise à élever les niveaux indiciaires des chargés de recherche pour les porter à ceux des maîtres assistants. En même temps, les conditions d'intégration pourraient être revues et la règle pratiquée pour les chercheurs du CNRS de l'intégration d'un chercheur dans le corps de l'enseignement supérieur à grade égal et non à l'échelon de début étendue aux autres instituts de recherche, au CEA notamment. Il va sans dire que la mise à l'étude d'un statut des personnels de l'Enseignement supérieur contraindra à aborder ces questions dans le détail. »

Il est intéressant de noter que le premier pas à faire à cette époque est d'abolir tout ce qui constitue plutôt une « sanction » à la mobilité qu'une incitation. Tel est le cas de ces dispositifs qui n'encourageaient pas les chercheurs à passer dans l'Enseignement supérieur. «La modification en cours des régimes de retraite, de manière à rapprocher, sur ce point, la situation des contractuels de celle des fonctionnaires titulaires aidera à supprimer les entraves à la mobilité, l'une des plus sensibles aux chercheurs. »

Le rapport du groupe de travail se termine ici. Rien n'est dit sur la politique que devraient mener les organismes de recherche pour faciliter, en l'état (1968) des statuts de leurs personnels respectifs, la mobilité inter-organismes. L'intérêt de ce document est qu'il donne une preuve claire que dans l'espace d'une trentaine d'année, depuis la fin de la guerre et la confirmation ou création des organismes de recherche, les différences se sont accentuées entre eux à tel point qu'un rapprochement paraît difficile, sinon impossible. Il n'est pas possible de traiter ici les discussions des années 1960 quand les directeurs des divers organismes de recherche venaient présenter et discuter avec les douze sages du CCSRST les problèmes qu'ils rencontraient au sein de leurs institutions. Les initiatives prises à la suite de ce rapport seraient aussi 
intéressantes à analyser. Une suggestion néanmoins peut être avancée en ce qui concerne les statuts de 1984. La titularisation dans la Fonction publique a été la solution « minimale » d'harmonisation des statuts des divers organismes et de l'Enseignement supérieur. Cela a permis d'abolir certains obstacles, comme celui du passage du statut de chercheur à celui d'enseignant-chercheur, sur la seule règle, imposée par le statut de la fonction publique, qu'un fonctionnaire doit être classé dans un indice égal ou immédiatement supérieur à celui qu'il occupait. Mais qu'en est-il de la mobilité, maintenant possible dans les faits, entre organismes et au sein d'un même organisme? C'est une étude qui reste à faire.

\section{NOTES}

1.Archives du CNRS, procès-verbal de la séance du conseil d'administration du 11 octobre 1946.

2.L'actuel Inserm.

3.Colloque de Caen, Les cahiers de la République, numéro spécial, janvier-février 1957.

4.Rapport sur la recherche fondamentale et l'enseignement scientifique supérieur, dans Colloque de Caen, op. cit.

5.Décret $n^{\circ}$ 59-1400 du 9 décembre 1959 fixant le statut du personnel chercheur du CNRS, Journal Officiel du 15 décembre 1959.

6.DGRST, Rapport du groupe de travail « Carrières des chercheurs et mobilité ", décembre 1968.

7.Il s'agit du SNCS, Syndicat national indépendant de la recherche scientifique (CGC) et de la section Recherche du Syndicat général de l'éducation nationale (sgen-CFDT).

8. Recherche et contestation. Débats des Travailleurs scientifiques après mai 1968, textes mis au point par L. Gatineau, A. Gerschel, L. Hay, R. Klapisch, P. Leroux-Hugon, J. Singer, G.

Vignaux, Paris, 1969.

\section{RÉSUMÉS}

What Kind of Status for the Researchers? The Debate in 1968

Since the beginning, the general administrators of the CNRS had to face with a very difficult question: could a researcher, which could benefit of only transitory position, remain until his retirement? This would mean that a new profession existed: researcher. At the conference on research organized in Caen, the question of a researcher's statute was debated. The two possibilities were: a) extend to researchers the model of the university's personnel, or adopt a new scheme adapted to the research constraints. In 1959 the first statute for CNRS researchers were stipulated. But, in 1968 the necessity of their modification was clearly perceived by the civil 
servants in the government. A committee was created to study the problem. Meanwhile, in May, universities and the CNRS were in ferment. Researchers organized a national meeting in July; one subject of discussion was the question of the statute for all the researchers in France. The commission appointed by the government had to consider the hostility of researchers and of the general administrators of the great majority of research institutions to their reform project.

INDEX

Mots-clés : statut, personnel, chercheurs, carrière

\section{AUTEUR}

\section{GIROLAMO RAMUNNI}

Girolamo Ramunni est professeur d'histoire des sciences au Cnam. Il est le rédacteur en chef de La Revue pour l'histoire du CNRS. Il est responsable de l'action concertée incitative «Cultures et sociétés dans le développement durable » auprès du ministère délégué à la Recherche. 